\title{
Wound Dressing Materials: The Essentials
}

\author{
Hyonsurk Kim
}

Department of Plastic and Reconstructive Surgery, Dankook University College of Medicine, Cheonan, Korea

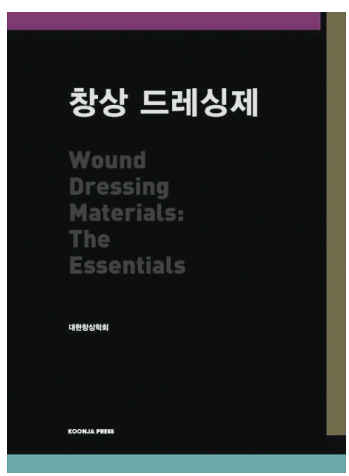

Korean Wound Management Society.

Wound Dressing Materials: The Essentials.

Print length: 259 pages.

Language: Korean

Koonja Publishing Inc.

Publication date: March 30, 2018

ISBN: 979-11-5955-285-4
According to the preface of Wound Dressing Materials: The Essentials, there are currently over 2,500 wound dressing-related products registered for use in the Republic of Korea. Since a large portion of these products are actually applied by clinicians who have not been trained or educated as wound care professionals, it falls upon the shoulders of those with expertise in wound care to help their colleagues in determining which kind of dressing is best suited to each different wound.

Wound Dressing Materials: The Essentials is a single-volume, 259-page long hardcover compilated by 22 wound experts of the Korean Wound Management Society including plastic surgeons, orthopedic surgeons, burn surgeons and wound, ostomy and continence nurses just for this purpose. Based on the contents of the Korean Wound Management Society's widely successful 2017 Educational Symposium, which was held in September 2017 with the theme "Wound Dressings - Where are we?", Wound Dressing Materials: The Essentials' objective is to provide down-to-earth knowledge and information concerning the choice and use of various dressing materials in real-life clinical settings of the Korean healthcare system - considering not only medical efficacy and safety but also patient comfort and financial compliance, National Health Insurance reimbursement regulations and documentation requirements.

Unlike some other publications on wound management, Wound Dressing Materials: The Essentials does not focus on complex theoretical studies or academic basic research, instead aiming to provide practical knowledge that can easily be implemented in clinical practice by presenting the clinical experiences of the authors backed by evidence-based literature reviews. An ample amount of photographs, figures and tables of the various dressing materials available in Korea referred to in the text, along with an abundance of case photographs, helps readers understand and apply the contents of this volume to their clinical practices with ease; the authors have put an emphasis

\author{
Book Review \\ Received: July 1, 2018 \\ Accepted: July 3, 2018 \\ Corresponding author: \\ Hyonsurk Kim, M.D. \\ Department of Plastic and Reconstructive \\ Surgery, Dankook University College of \\ Medicine, 201 Manghyang-ro, Dongnam-gu, \\ Cheonan 31116, Korea \\ Tel: +82-41-550-6285 \\ Fax +82-41-556-0524 \\ E-mail: kruezel@gmail.com
}

This is an Open Access article distributed under the terms of the Creative Commons Attribution Non-Commercial License (http://creativecommons.org/licenses/by-nc/4.0/) which permits unrestricted non-commercial use, distribution, and reproduction in any medium, provided the original work is properly cited.

(c) 2018 Korean Wound Management Society 
on ensuring that their book can readily be understood even by clinicians not familiar to the complexities of wound management.

The book is composed of 3 parts. Part I, with 5 chapters, constitutes an overview of wounds and wound dressings. Classification of dressing types, classification of dressing products and clinical dressing practice guidelines are included in Part I. Of particular interest is Chapter 5, 'Reimbursement and Documentation', which provides crucial information on how much of each dressing type is permitted under what kind of circumstances by Korea's nationwide Health Insurance system, and what documentation is required as legal proof for reimbursement of such dressings - contents that many tertiary medical institute-based wound care physicians are strangely ignorant of. Part II covers the clinical characteristics and use of actual dressing materials, from simple gauze to state-of-the-art composite and biologic dressings, over 9 chapters. Part III offers some special considerations for dressings: Chapter 15, Dressing-Related Skin Injury, Chapter 16, Dressing-Related Pain, and Chapter 17, Accessories for Dressing, the contents of which are sadly ignored by many clinicians but definitely must be kept in mind at all times to prevent wound exacerbation and ensure patient welfare.

With an easy-to-read concise format, bountiful graphic depictions and solid substance, Wound Dressing Materials: The Essentials provides much more than its price value to a wide spectrum of clinicians engaging in wound care. I would readily recommend it to any nurse or physician who frequently finds him- or herself in the position of choosing the best dressing material for a certain wound, both for building a basic foundation of dressing principles and updating wound management skills.

\section{Conflict of interest}

No potential conflicts of interest relevant to this article were reported. 